\title{
Roles of microRNA-186 and vascular endothelial growth factor in hepatocellular carcinoma complicated with portal vein tumor thrombus
}

\author{
WEIDONG YUAN $^{1^{*}}$ and FUGUANG LI ${ }^{2 *}$ \\ ${ }^{1}$ Department of Hepatobiliary Surgery, The Affiliated Huai'an No. 1 People's Hospital of Nanjing Medical University, \\ Huai'an, Jiangsu 223300; ${ }^{2}$ The Second Ward of General Surgery Department, \\ Ankang City Central Hospital, Ankang, Shaanxi 725000, P.R. China
}

Received April 6, 2016; Accepted June 26, 2018

DOI: $10.3892 /$ etm.2020.9092

\begin{abstract}
The present study aims to investigate the role and underlying mechanism of microRNA (miR)-186 in patients with hepatocellular carcinoma (HCC) complicated with portal vein tumor thrombus. Blood samples from $29 \mathrm{HCC}$ patients with portal vein tumor thrombus were collected between January 2014 and September 2015 in Huai'an First People's Hospital, while blood from $36 \mathrm{HCC}$ patients without vein tumor thrombus was also collected in the same period. In addition, tumor thrombus specimens were collected from the HCC patients with portal vein tumor thrombus, and peritumoral tissues of the tumor thrombus were used as the control. Reverse transcription-quantitative polymerase chain reaction, ELISA and western blot analyses were applied to detect vascular endothelial growth factor (VEGF) expression at the mRNA and protein levels. Bioinformatics prediction was used to predict the target of miR-186, and then miR-186 expression was detected. Furthermore, dual-luciferase reporter assay was used to validate whether miR-186 directly targeted VEGF. Following transfection with agomiR-186, the expression levels of miR-186 and VEGF were detected, while MTT assay was used to detect EA.hy926 cell proliferation subsequent to small interfering RNA (siRNA) silencing. The results identified that VEGF was significantly increased in the tumor thrombus and blood samples of HCC patients with vein tumor thrombus at the mRNA and protein levels, while miR-186 expression was significantly decreased $(\mathrm{P}<0.05)$. Following silencing VEGF
\end{abstract}

Correspondence to: Dr Weidong Yuan, Department of Hepatobiliary Surgery, The Affiliated Huai'an No. 1 People's Hospital of Nanjing Medical University, 6 Beijing West Road, Huai'an, Jiangsu 223300, P.R. China

E-mail:vnm555@126.com

${ }^{*}$ Contributed equally

Key words: microRNA-186, hepatocellular carcinoma, portal vein tumor thrombus by siRNA transfection, the proliferation of EA.hy926 cells was inhibited. In addition, VEGF expression was significantly decreased and cell proliferation was reduced when upregulating miR-186. Dual-luciferase reporter assay demonstrated that miR-186 regulated VEGF expression through complementary binding to 3'-untranslated region. In conclusion, VEGF was significantly increased in tumor thrombus and blood samples from HCC patients with vein tumor thrombus, which may be associated with the downregulation of miR-186. Thus, miR-186 may promote the development and progression of vein tumor thrombus in HCC.

\section{Introduction}

Hepatocellular carcinoma (HCC) is one of common types of gastrointestinal cancer with high rates of recurrence and metastasis in the liver, and the overall prognosis for patients is poor (1-3). The 5-year survival rate of HCC is $\sim 32.5 \%$ in China, and only $\sim 59.1 \%$ in patients diagnosed at an early stage $(4,5)$. In Western countries, the 5-year survival rate has been reported to be $<12 \%(4,5)$. In 1869 , Armand Trousseau observed that cancer patients may be complicated by venous thrombosis, and this is known as Trousseau syndrome (6). One of the high risk factors for induction of venous thrombosis has been demonstrated to be cancer, and the incidence rate of venous thrombosis reached $\sim 12.3 \%$ for cancer patients following diagnosis (7). However, the mechanisms underlying tumor thrombus induced by malignant tumor remain unclear.

It has been observed that numerous microRNAs (miRs) and mRNAs are involved in Trousseau syndrome (8). Different factors, including integrin $\beta 3$ (9) and nuclear factor- $\kappa \mathrm{B}(10)$, participate in the processes. Similar to the development of tumor, the induction of tumor thrombus is closely associated with angiogenesis (11). Recent studies mainly focused on the roles and signaling transductions associated with vascular endothelial growth factor (VEGF) in the progression of tumor thrombus (12-14). The excessive growth of blood vessels is one of the important conditions for the formation of tumors and tumor thrombi (15-17), therefore the EA.hy926 cell line was used in the current study. 
In the present study, VEGF expression was detected at the mRNA and protein levels in blood and tumor thrombus specimens collected from HCC patients complicated with vein tumor thrombus. The association between miR-186 and VEGF expression levels was also validated.

\section{Materials and methods}

Sample collection. The current study included 29 cases of HCC patients with portal vein tumor thrombus, whose tumor thrombus was resected between January 2013 and September 2015 in the Department of Hepatobiliary Surgery of Huai'an First People's Hospital (Huai'an, China), and the peritumoral tissues of the tumor thrombus were also collected as control tissues. During the same period, blood samples from 39 HCC patients without tumor thrombus were used as the control group. There were 18 males and 11 females among the HCC patients with portal vein tumor thrombus, and the age range was between 26 and 66 years, with a median age of 45.6 years. In the control group without tumor thrombus, there were 26 males and 13 females, and their age ranged between 21 and 77 years, with a median age of 49.5 years. All the included patients presented first-onset $\mathrm{HCC}$, and had not received hormone therapy, medication, radiotherapy and chemotherapy prior to inclusion. All the patients were diagnosed by pathologists at the Huai'an First People's Hospital (18). The portal vein tumor thrombus was confirmed by ultrasound or based on the symptoms, signs and diagnosis history. The resected tumor thrombus and peritumoral tissues were stored at $-80^{\circ} \mathrm{C}$ within $2 \mathrm{~h}$. Fasting peripheral blood samples were collected from all patients upon diagnosis and stored at $-20^{\circ} \mathrm{C}$ following addition of EDTA anticoagulant. Prior written and informed consent was obtained from every patient, and the study was approved by the Ethics Review Board of Nanjing Medical University (Huai'an, China).

Reverse transcription-quantitative polymerase chain reaction $(R T-q P C R)$. Total RNA from blood and tissue samples was extracted by TRIzol reagent (Invitrogen; Thermo Fisher Scientific, Inc., Waltham, MA, USA). The RNA quality of samples was checked by $1 \%$ gel electrophoresis and the absorbance ratio at 260/280 nm was detected by a spectrophotometer. The cDNA was obtained with the TIANScript II cDNA first strand synthesis kit (cat. no. KR107; Tiangen Biotech Co., Ltd., Beijing, China). The SuperReal PreMix (SYBR Green) kit (cat. no. FP204; Tiangen Biotech Co., Ltd.) was used to detect the mRNA expression of VEGF, with $\beta$-actin used as the internal reference. Primers used in qPCR were as follows: VEGF forward, 5'-TTGCCTTGCTGCTCTACCTC-3', and reverse, 5'-AAA TGCTTTCTCCGCTCTGA-3'; $\beta$-actin forward, 5'-TGACGT GGACATCCGCAAAG-3', and reverse, 5'-CTGGAAGGT GGACAGCGAGG-3'. The reaction was performed in a $25-\mu 1$ system, including $10 \mu \mathrm{l}$ SuperReal PreMix (SYBR Green), $1 \mu \mathrm{l}$ forward primer, $1 \mu \mathrm{l}$ reverse primer, $2 \mu \mathrm{l}$ cDNA template

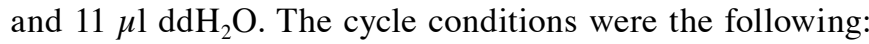
$94^{\circ} \mathrm{C}$ for $2 \mathrm{~min}$, followed by 35 cycles of $94^{\circ} \mathrm{C}$ for $30 \mathrm{sec}$, $55^{\circ} \mathrm{C}$ for $30 \mathrm{sec}, 71^{\circ} \mathrm{C}$ for $1 \mathrm{~min}$ and a further $71^{\circ} \mathrm{C}$ for $2 \mathrm{~min}$. Relative expression of VEGF against $\beta$-actin was calculated by the $2^{-\Delta \Delta \mathrm{Cq}}$ method (19).
Reverse transcription of miRNA followed the procedure provided by the manufacturer of the miRcute miRNA cDNA kit (KR201; Tiangen Biotech Co., Ltd.). qPCR was then used to detect the expression of miR-186, with U6 used as an internal reference. The primers were as follows: miR-186 forward, 5'-CCCGATAAAGCTAGATAACC-3', and reverse, 5'-CAGTGCGTGTCGTGGAGT-3'; U6 forward, 5'-GCTTCG GCAGCACATATACTAAAAT-3', and reverse, 5'-CGCTTC ACGAATTTGCGTGTCAT-3'. The reaction was conducted in a $25-\mu 1$ system, including $10 \mu 1$ SuperReal PreMix (SYBR Green), $1 \mu \mathrm{l}$ forward primer, $1 \mu 1$ reverse primer, $2 \mu \mathrm{l}$ cDNA template and $11 \mu \mathrm{l} \mathrm{ddH}_{2} \mathrm{O}$. The thermal cycling conditions were the following: $95^{\circ} \mathrm{C}$ for $5 \mathrm{~min}$, followed by 40 cycles of $95^{\circ} \mathrm{C}$ for $10 \mathrm{sec}, 60^{\circ} \mathrm{C}$ for $20 \mathrm{sec}$ and $72^{\circ} \mathrm{C}$ for $20 \mathrm{sec}$. The relative expression of miR-186/U6 was calculated by the $2^{-\Delta \Delta \mathrm{Cq}}$ method.

ELISA. The peripheral blood was centrifuged at $1,000 \mathrm{x} \mathrm{g}$ at $4^{\circ} \mathrm{C}$ for $10 \mathrm{~min}$ to separate the serum and red blood cells, and the serum was used for subsequent analysis. ELISA was performed according to the instructions provided by the human VEGFA ELISA kit (cat. no. 100663; Abcam, Cambridge, MA, USA). Next, 50- $\mu$ l standard were added into different wells on the ELISA plate, with $10 \mu \mathrm{l}$ sample and $40 \mu \mathrm{l}$ dilution buffer added into each well. With the exception of blank wells, $100 \mu \mathrm{l}$ horseradish peroxidase (HRP)-labeled antibody was added to each well, covered with microplate sealers and incubated for $1 \mathrm{~h}$. Subsequent to five washes with the washing solution in the kit $50 \mu 1$ substrate $\mathrm{A}$ and $50 \mu \mathrm{l}$ substrate $\mathrm{B}$ were added into each well. After incubation at $37^{\circ} \mathrm{C}$ for $15 \mathrm{~min}, 50 \mu \mathrm{l}$ stop solution was added into each well. The optical density value was measured at a wavelength of $450 \mathrm{~nm}$ within $15 \mathrm{~min}$.

Bioinformatics prediction. Bioinformatics prediction is the basis to explore the functions of miRNAs. The miRanda (http://www.microma.org/rnicroma/home. do), TargetSean (www.targetscan.org), PiTa (http://genie. weizmann.ac.il/pubs/mir07/mir07_data.html), RNAhybrid (http://bibiserv.techfak.uni-bielefeld.de/rnahybrid/) and PICTA (http://pictar.mdc-berlin.de/) databases were used in the present study to predict the potential miRNAs that can directly regulate VEGF physiologically and in pathological conditions, according to previously described methods (20-22). Based on the results of bioinformatics analysis, miR-186 was selected as a miRNA regulating VEGF expression. The authors of the current study investigated miR-186 previously. Additionally, previous reports have demonstrated that miR-186 could act as tumor suppressor $(23,24)$; therefore, miR-186 was selected for analysis in the current study.

Dual-luciferase reporter assay. Based on the bioinformatics prediction, the wild-type 3'-untranslated region (UTR) and the mutant 3'-UTR of VEGF were synthesized in vitro, and were cloned into the downstream of pMIR-REPORT luciferase vector (Ambion; Thermo Fisher Scientific, Inc.) by Spe-1 and HindIII enzyme. 293 T cells were co-transfected with agomiR-186 (100 nM; Sangon Biotech Co., Ltd., Shanghai, China) mimics and with wild-type VEGF 3'-UTR or mutant 3'-UTR. Following transfection with Lipofectamine 2000 
reagent (cat. no. 11668-027; Thermo Fisher Scientific, Inc.) for $24 \mathrm{~h}$, cells were lysed and luciferase intensity was measured by GloMax 20/20 luminometer (Promega Corp., Madison, WI, USA) based on the standard protocol of the luciferase kit (cat. no. E1910; Promega Corp.). The intensity of Renilla was used as a control, and the fluorescence intensity in the NC, wild-type and mutant groups was analyzed.

Cell transfection. At $24 \mathrm{~h}$ prior to transfection, $3 \times 10^{5}$ logarithm growth EA.hy926 cells (Type Culture Collection of the Chinese Academy of Sciences, Shanghai, China) were seeded in 24-well plate and cultured into antibiotics-free F12/Dulbecco's modified Eagle's medium (DMEM) containing 10\% fetal bovine serum. When cells reached $\sim 70 \%$ confluence, transfection was performed. A total of $1 \mu \mathrm{g} / \mu \mathrm{l}$ plasmid, $30 \mathrm{nM}$ VEGF small interfering RNA (siRNA; target sequence, 106-AATCAT CACGAAGTGGTGAAGTT; forward, 5'-UCAUCACGAAGU GGUGAAGdTdT-3' and reverse 5'-CUUCACCACUUCGUG AUGAdTdT-3') or $30 \mathrm{nM}$ agomiR-186 (forward, 5'-CAAAGA AUUCUCCUUUUGGGCU-3' and reverse 5'-GCCCAAAGG UGAAUUUUUUGGG-3'; all Sangon Biotech Co., Ltd.) and Lipofectamine 2000 were added into EP tubes containing $50 \mu 1$ DMEM, respectively. The two tubes were mixed together after 5 -min incubation at room temperature, and the mixture was added to each well after incubation for $20 \mathrm{~min}$ at room temperature. After transfection for $48 \mathrm{~h}$, the cells were collected to detect the expression of VEGF at the mRNA and protein levels.

Western blot analysis. Total proteins were extracted by protein lysis using RIPA buffer (P0013B; Beyotime Institute of Biotechnology, Haimen, China) based on a standard protocol, and the protein concentration was detected by a BCA assay kit (Zhongke Ruitai Biotechnology Co., Ltd., Beijing, China). After boiling with loading buffer for $5 \mathrm{~min}, 20 \mu \mathrm{g}$ protein was subjected to $10 \%$ SDS-PAGE and then transferred to a polyvinylidene fluoride membrane under ice bath (constant voltage of $100 \mathrm{~V}$ for $2 \mathrm{~h}$ ). Subsequent to blocking by $5 \%$ skim milk, rabbit anti-human polyclonal VEGF $(1: 1,000$; cat. no. ab46154) and rabbit anti-human $\beta$-actin $(1: 5,000$; cat. no. ab129348) primary antibodies were added at $4^{\circ} \mathrm{C}$ overnight. Next, HRP-conjugated goat anti-rabbit IgG secondary antibody (1:3,000; cat. no. ab6721) was added at $37^{\circ} \mathrm{C}$ for $1 \mathrm{~h}$. All the antibodies were purchased from Abcam. Finally, the membrane was developed by the BeyoECL Plus enhanced chemiluminescence reagent (P0018; Beyotime Institute of Biotechnology). The developed film was scanned and analyzed by Image Lab 3.0 software (Bio-Rad Laboratories Inc., Hercules, CA, USA). $\beta$-actin was used as an internal control to calculate the relative expression of VEGF.

MTT assay. EA.hy926 cells were seeded in 96-well plates with $2 \times 10^{3}$ cells/well, and each sample had three replicates. At 24, 48 and 72 h, $20 \mu \mathrm{l}$ MTT (5 g/l) was added into each well, followed by addition of $150 \mu \mathrm{l}$ dimethyl sulfoxide to resolve the purple crystals. After incubation for $4 \mathrm{~h}$ at $37^{\circ} \mathrm{C}$, the absorbance of cells was measured at $490 \mathrm{~nm}$ wavelength and the proliferation curves were plotted.

Statistical analysis. The SPSS version 18.0 software (SPSS, Inc., Chicago, IL, USA) was used to perform statistical analysis.
All the data are presented as the mean \pm standard deviation, and normality test was used. One-way analysis of variance was used to compare difference among multiple groups. When variance was homogenous, the least significant difference and Student-Newman-Keuls methods were used; otherwise, Tamhane's T2 or T3 methods were applied. $\mathrm{P}<0.05$ was considered as an indicator of statistically significant differences.

\section{Results}

Expression of VEGF mRNA in tumor thrombus and blood samples. To examine the expression changes between tumor thrombus and peritumoral tissues in HCC patients with portal vein tumor thrombus, RT-qPCR was conducted to detect VEGF mRNA. Compared with peritumoral tissues, VEGF mRNA expression was significantly increased in the tumor thrombus $(\mathrm{P}<0.01$; Fig. 1A). Similar to the results in tumor tissues, VEGF mRNA expression was also significantly increased in the blood of patients with portal vein tumor thrombus when compared with that in the blood of patients without tumor thrombus $(\mathrm{P}<0.01$; Fig. 1B). The results indicate that VEGF may serve regulatory roles in the development of tumor thrombus in HCC.

Expression of VEGF protein in tumor thrombus and blood. To validate the expression of VEGF protein in the tumor thrombus tissues and blood samples in HCC patients with tumor thrombus, western blot analysis and ELISA were used, respectively. Compared with the control group, VEGF protein was significantly increased in the tumor thrombus tissues and blood of patients with portal vein tumor thrombus $(\mathrm{P}<0.01$; Fig. 2). The results revealed that VEGF protein expression was consistent with VEGF mRNA expression, which further indicates that VEGF may be involved in the development of tumor thrombus in HCC patients.

Expression of miR-186 in tumor thrombus and blood. In order to investigate the roles of miR-186 in tumor thrombus in HCC patients, RT-qPCR was used to detect its expression in tumor thrombus tissue and blood samples. As shown in Fig. 3, miR-186 expression was significantly downregulated both in the tumor thrombus tissues and in the blood, when compared with the control samples $(\mathrm{P}<0.05)$. This result indicates that miR-186 may serve a role in the development of tumor thrombus in HCC patients.

VEGF is directly targeted by miR-186. To determine whether VEGF was directly targeted by miR-186, bioinformatics prediction was performed. The miR-186 was predicted as one of the miRNAs that regulates VEGF. The complementary binding site of miR-186 with VEGF is shown in Fig. 4A. To further verify this result, the dual-luciferase reporter assay was conducted. As shown in Fig. 4B, the fluorescence intensity was significantly downregulated in $293 \mathrm{~T}$ cells co-transfected with agomiR-186 and pMIR-REPORT-wild type plasmid in comparison with the negative control group $(\mathrm{P}<0.01)$. By contrast, there was no significant difference between cells co-transfected with agomiR-186 and pMIR-REPORT-mutant in comparison with the negative control group $(\mathrm{P}>0.05)$. These results indicate that miR-186 can regulate VEGF expression through complementary binding to the 3'-UTR of VEGF mRNA. 

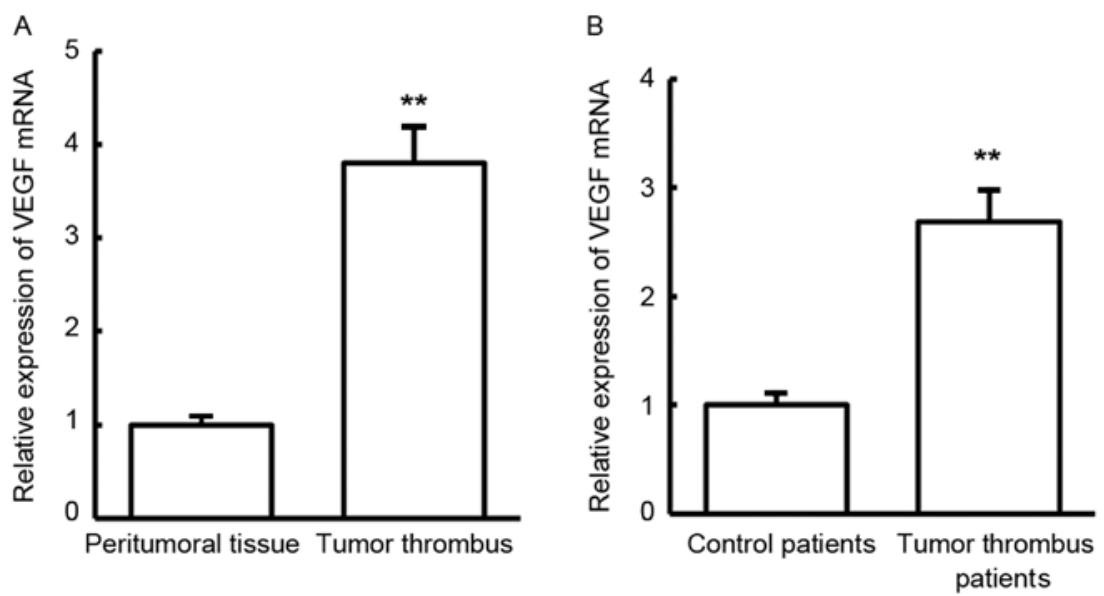

Figure 1. VEGF mRNA expression in tumor thrombus tissue and blood of HCC patients with portal vein tumor thrombus. (A) Compared with peritumoral tissues, the expression of VEGF mRNA was significantly increased in tumor thrombus tissues. (B) Compared with blood from HCC patients without tumor thrombus, the expression of VEGF mRNA was significantly increased in the blood of HCC patients with portal vein tumor thrombus. ${ }^{* *} \mathrm{P}<0.01 \mathrm{vs}$. control group. HCC, hepatocellular carcinoma; VEGF, vascular endothelial growth factor.

Effects of miR-186 on proliferation of EA.hy926 cells. To analyze the influence of miR-186 on cell proliferation, MTT assay was performed. As shown in Fig. 5A, miR-186 expression was significantly increased in EA.hy926 cells subsequent to transfection with agomiR-186 $(\mathrm{P}<0.01)$. At the same time, as shown in Fig. 5B, VEGF expression was significantly decreased in EA.hy926 cells following transfection with agomiR-186 $(\mathrm{P}<0.01)$. Compared with the control group, EA.hy926 cell proliferation was significantly inhibited in cells after transfection with agomiR-186 ( $<<0.05$; Fig. 5C). These results suggest that miR-186 may inhibit the proliferation of EA.hy926 cells through regulating VEGF expression.

Effects of VEGF siRNA on proliferation of EA.hy926 cells. To further validate the aforementioned results indicating that EA.hy 926 cell proliferation was inhibited by miR-186 through downregulation of VEGF, a siRNA was used to downregulate VEGF expression and then cell proliferation was detected by MTT assay. As shown in Fig. 6A and B, VEGF expression at the mRNA and protein levels was significantly decreased following transfection with VEGF siRNA in EA.hy926 cells $(\mathrm{P}<0.05)$. In addition, the MTT results revealed that cell proliferation was significantly reduced in the agomiR-186 transfection group (Fig. 6C). The results further confirm that downregulated VEGF may inhibit the proliferation of EA.hy926 cells.

\section{Discussion}

In the present study, VEGF expression was detected in the blood and tumor thrombus tissues of HCC patients with portal vein tumor thrombus, and the expression of upstream regulator miR-186 was analyzed. Through cell experiments, the functions of miR-186 and VEGF were preliminary discussed, and then the underlying molecular mechanisms were examined.

Vein tumor thrombus is a common complication in malignant process and the second most common cause of patient mortality besides cancer itself (25). Approximately $10 \%$ of patients with renal cell cancer will develop venous tumor thrombus $(26,27)$, and $60 \%$ of patients with tumor thrombus are associated with metastasis (28). For patients with tumor thrombus, complete resection of the tumor is a treatment method (26). Currently, blocking cell signaling pathways and angiogenesis in tumors to inhibit tumor growth is one of the efficient targeted therapies to treat cancer. Certain studies have reported that targeted therapies are a new adjuvant treatment for tumor thrombus (29-33). In normal environment, VEGF is one of the most effective pro-angiogenic factors and regulators of blood vessel growth. Its basic function is to promote angiogenesis and increase blood supply (34). It has also been reported that VEGF is an important factor in HCC (35). The EA.hy926 cell line was selected for the current study based on previous studies (15-17). In line with previous findings, the present study observed that VEGF was abnormally expressed in the blood and tumor thrombus tissues of HCC patients with portal vein tumor thrombus, whereas silencing VEGF slowed down the proliferation of EA.hy926 endothelial cells. All these results indicate that VEGF may serve an important role in the development of tumor thrombus in HCC patients.

To further investigate the regulatory mechanisms of VEGF, the upstream regulators of VEGF were predicted through bioinformatics methods. miRNAs may inhibit mRNA translation through degrading mRNA (36). In fact, these regulatory mechanisms of miRNAs to upregulate or downregulate several genes serve important roles in the development and progression of tumors $(37,38)$. According to bioinformatics prediction, the current study identified that miR-186 may be an upstream miRNA to regulate VEGF.

Several studies have demonstrated that miR-186 may be a novel target in tumor prevention, diagnosis and treatment. For instance, Zhang et al (39) found that miR-186 can be used as a diagnostic biomarker in pancreatic cancer, while it also influenced proliferation and invasion of tumor cells. In addition, Lee et al (40) demonstrated that upregulated miR-186 was associated with recession of fibroblast proliferation. Sun et al (41) also observed that miR-186 participated in the formation of fibroblasts in tumors. Furthermore, a study by Cui et al (42) demonstrated that miR-186 inhibited cell 

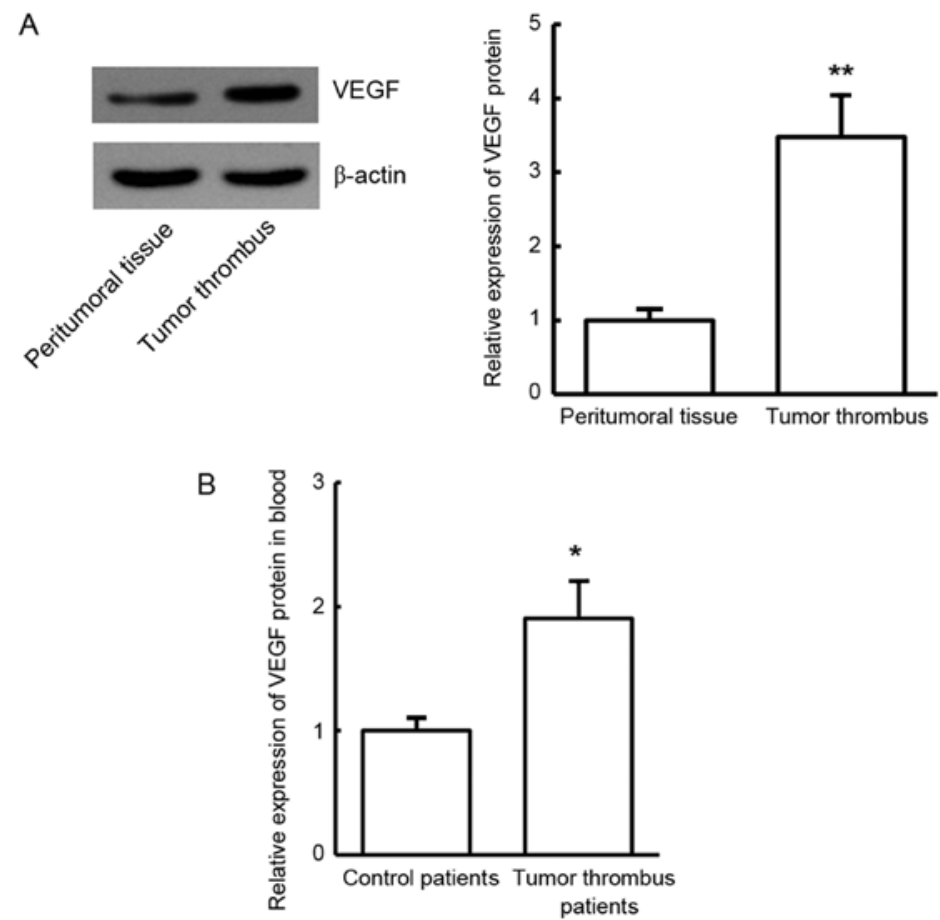

Figure 2. VEGF protein expression in tumor thrombus tissues and blood of HCC patients with portal vein tumor thrombus. (A) Western blot analysis indicated that, compared with peritumoral tissues, the expression of VEGF protein was significantly increased in tumor thrombus tissues. (B) ELISA indicated that, compared with blood from HCC patients without tumor thrombus, the expression of VEGF protein was significantly increased in the blood of HCC patients with portal vein tumor thrombus. ${ }^{*} \mathrm{P}<0.05$ and $^{* *} \mathrm{P}<0.01$ vs. control group. HCC, hepatocellular carcinoma; VEGF, vascular endothelial growth factor.
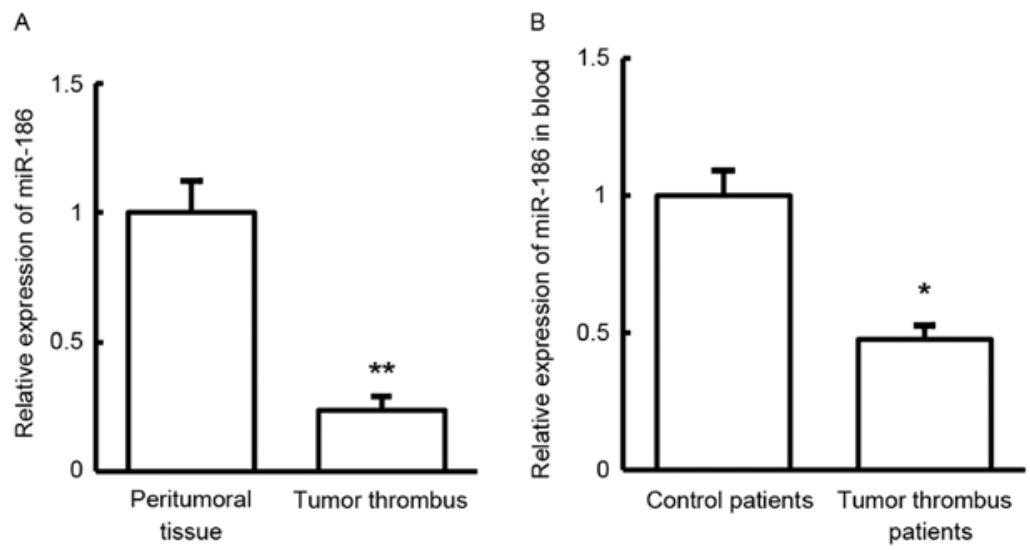

Figure 3. miR-186 expression in tumor thrombus tissues and blood of HCC patients with portal vein tumor thrombus. miR-186 expression was assessed by reverse transcription-quantitative polymerase chain reaction analysis. (A) Compared with peritumoral tissues, the expression of miR-186 was significantly decreased in tumor thrombus tissues. (B) Compared with the blood of HCC patients without tumor thrombus, miR-186 expression was significantly increased in the blood of HCC patients with portal vein tumor thrombus. " $\mathrm{P}<0.05$ and ${ }^{* *} \mathrm{P}<0.01$ vs. control group. HCC, hepatocellular carcinoma; miR, microRNA.

A

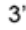

410:5
ucgggUUUUCCUCU-UAAGAAAC 5' hsa-miR-186 III I I IIIIIII auauaAAAUAUAUAUAUUCUUUU 3 ' VEgFA

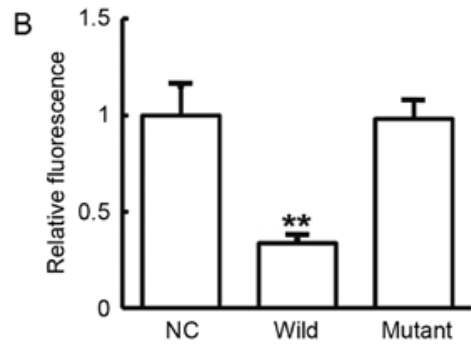

Figure 4. Analysis of VEGF regulation by miR-186. (A) The 'seed' region of miR-186 and complementary binding site in the 3'-UTR of VEGF mRNA. (B) Dual-luciferase reporter assay was used to detect whether VEGF is directly targeted by miR-186. Compared with the NC group, fluorescence was significantly lower in cells transfected with the wild-type VEGF 3'-UTR plasmid. ${ }^{* *} \mathrm{P}<0.01$ vs. control group. VEGF, vascular endothelial growth factor; miR, microRNA; UTR, untranslated region; NC, normal control. 
A

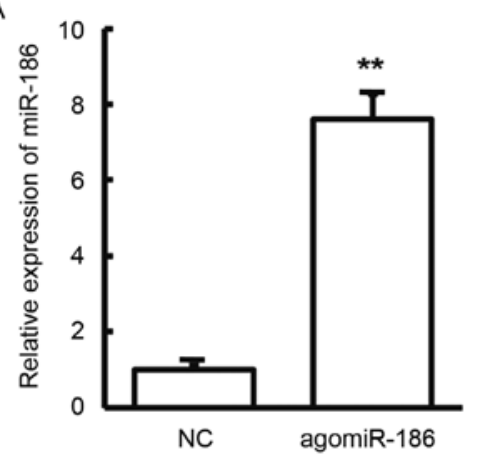

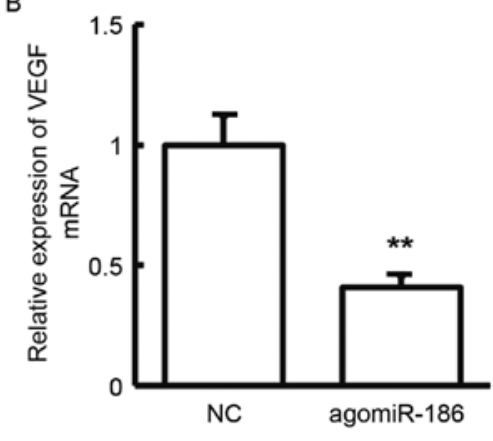

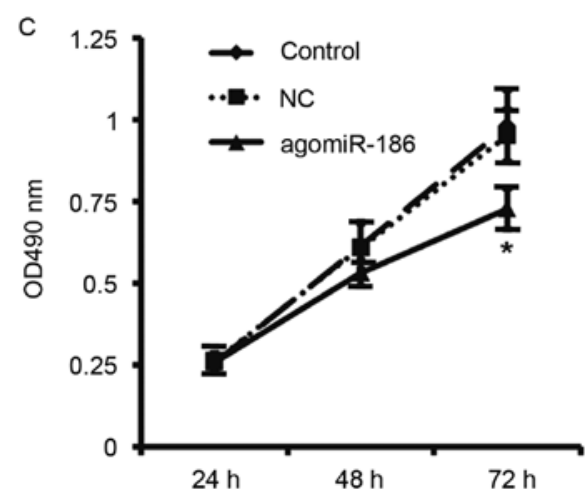

Figure 5. Effect of transfection with agomiR-186 on EA.hy926 cell proliferation. Reverse transcription-quantitative polymerase chain reaction analysis demonstrated that (A) miR-186 expression was significantly increased and (B) VEGF mRNA expression was significantly decreased in EA.hy926 cells following transfection with agomiR-186. (C) The MTT assay revealed that, compared with the control group, the proliferation was significantly inhibited in the agomiR-186 transfection group. ${ }^{*} \mathrm{P}<0.05$ and ${ }^{* *} \mathrm{P}<0.01$ vs. control group. VEGF, vascular endothelial growth factor; miR, microRNA; NC, normal control.

A

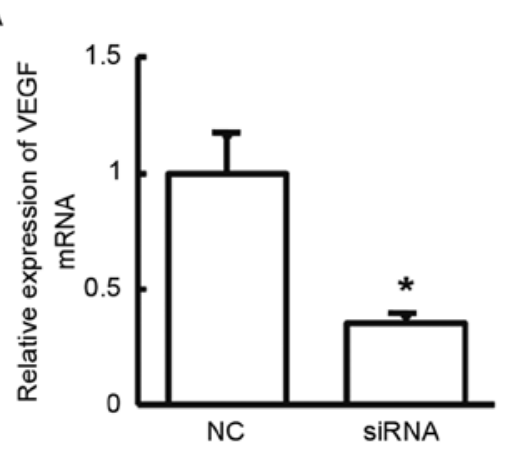

B

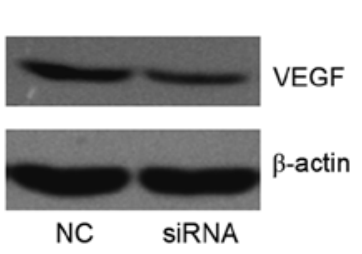

NC SiRNA

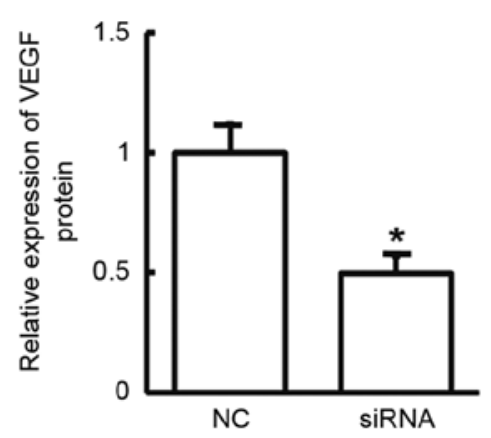

C

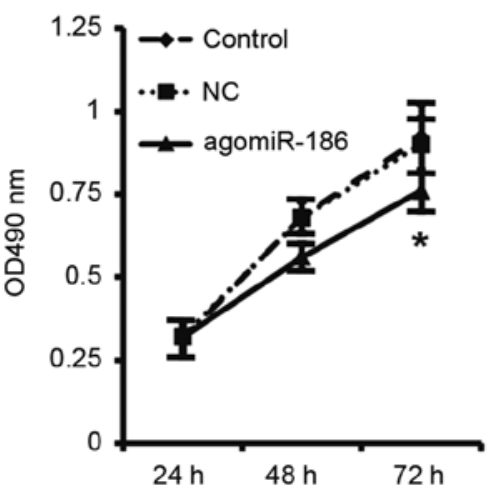

Figure 6. Effect of transfection with VEGF siRNA on EA.hy926 cell proliferation. (A) Reverse transcription-quantitative polymerase chain reaction analysis demonstrated that VEGF mRNA expression was significantly decreased in EA.hy926 cells following transfection with VEGF siRNA. (B) Western blot analysis demonstrated that VEGF protein expression was significantly decreased in EA.hy926 cells following transfection with VEGF siRNA. (C) Compared with the control group, the proliferation was significantly inhibited in the VEGF siRNA transfection group, as detected by a MTT assay. ${ }^{*} \mathrm{P}<0.05$ vs. control group. VEGF, vascular endothelial growth factor; siRNA, small interfering RNA; NC, normal control; OD, optical density. 
proliferation and metastasis in non-small cell lung cancer through regulating Rock1. To further examine the molecular mechanism underlying the action of miR-186 on VEGF, human endothelial cell EA.hy926 were cultured in vitro and transfected with agomiR-186 to analyze the alterations in cell proliferation by MTT assay. The results indicated that miR-186 reduced the proliferation of EA.hy926 cells, while upregulated miR-186 induced the downregulation of VEGF. In addition, dual-luciferase reporter assay confirmed that miR-186 was able to directly bind to the 3'-UTR of VEGF mRNA, suggesting that miR-186 may regulate VEGF mRNA expression.

In conclusion, the findings of the present study suggested that miR-186 serves important roles in the development of portal vein tumor thrombus in HCC through regulating VEGF expression. miR-186 may be used as a target for diagnosis, prevention and treatment of HCC patients with tumor thrombus. The current findings provide novel insight for understanding the development and progression of tumor thrombus in HCC patients.

\section{Acknowledgements}

The authors would like to thank Professor Dianhua Gu (Department of Hepatobiliary Surgery, Huai'an First People's Hospital, Nanjing Medical University, Huai'an, China) for his valuable help during the preparation of this manuscript.

\section{Funding}

No funding was received.

\section{Availability of data and materials}

All data generated or analyzed during this study are included in this published article.

\section{Authors' contributions}

WDY designed the study and performed the experiments. FGL designed the study, performed the statistical analysis and prepared the manuscript.

\section{Ethics approval and consent to participate}

Not applicable.

\section{Patient consent for publication}

Not applicable.

\section{Competing interests}

The authors declare that they have no competing interests.

\section{References}

1. Kanda M, Sugimoto H and Kodera Y: Genetic and epigenetic aspects of initiation and progression of hepatocellular carcinoma. World J Gastroenterol 21: 10584-10597, 2015.

2. Kuszyk BS, Beauchamp NJ Jr and Fishman EK: Neurovascular applications of CT angiography. Semin Ultrasound CT MR 19: 394-404, 1998
3. Wedd JP, Nordstrom E, Nydam T, Durham J, Zimmerman M, Johnson T, Thomas Purcell W and Biggins SW: Hepatocellular carcinoma in patients listed for liver transplantation: Current and future allocation policy and management strategies for the individual patient. Liver Transpl 21: 1543-1552, 2015.

4. Yang BH, Xia JL, Huang LW, Tang ZY, Chen MS, Li JQ Liang AM, Mo QG, Lu HS, Dai CL, et al: Changes of clinical aspect of primary liver cancer in China during the past 30 years-control study for 3,250 cases with primary liver cancer. Zhonghua Yi Xue Za Zhi 83: 1053-1057, 2003 (In Chinese).

5. El-Serag HB: Hepatocellular carcinoma. N Engl J Med 365: 1118-1127, 2011.

6. Trousseau A: Lectures on clinical medicine, delivered at the Hotel-Dieu, Paris: Translated and edited with notes and appendices, by P. Victor Bazire. Lindsay and Blakiston, 1869.

7. Blom JW, Vanderschoot JP, Oostindiër MJ, Osanto S, van der Meer FJ and Rosendaal FR: Incidence of venous thrombosis in a large cohort of 66,329 cancer patients: Results of a record linkage study. J Thromb Haemost 4: 529-535, 2006.

8. Meyer G: Venous thromboembolism and cancer. Rev Prat 65: 216-219, 2015 (In French)

9. Bianconi D, Schuler A, Pausz C, Geroldinger A, Kaider A, Lenz HJ, Kornek G, Scheithauer W,Zielinski CC, Pabinger I, et al: Integrin beta-3 genetic variants and risk of venous thromboembolism in colorectal cancer patients. Thromb Res 136: 865-869, 2015.

10. Malaponte G, Signorelli SS, Bevelacqua V, Polesel J, Taborelli M, Guarneri C, Fenga C, Umezawa K and Libra M: Increased levels of NF-kB-dependent markers in cancer-associated deep venous thrombosis. PLoS one 10: e0132496, 2015.

11. Connolly GC, Phipps RP and Francis CW: Platelets and cancer-associated thrombosis. Semin Oncol 41: 302-310, 2014.

12. Chang LH, Pan SL, Lai CY, Tsai AC and Teng CM: Activated PAR-2 regulates pancreatic cancer progression through ILK/HIF- $\alpha$-induced TGF- $\alpha$ expression and MEK/VEGF-A-mediated angiogenesis. Am J Pathol 183: 566-575, 2013

13. Lee $S$ and Goldfinger LE: RLIP76 regulates HIF-1 activity, VEGF expression and secretion in tumor cells, and secretome transactivation of endothelial cells. FASEB J 28: 4158-4168, 2014.

14. Posch F, Thaler J, Zlabinger GJ, Königsbrügge O, Koder S, Zielinski C, Pabinger I and Ay C: Soluble Vascular Endothelial Growth Factor (sVEGF) and the risk of venous thromboembolism in patients with cancer: Results from the Vienna Cancer and Thrombosis Study (CATS). Clin Cancer Res 22: 200-206, 2016.

15. Arutyunyan I, Fatkhudinov T, Kananykhina E, Usman N, Elchaninov A, Makarov A, Bolshakova G, Goldshtein D and Sukhikh G: Role of VEGF-A in angiogenesis promoted by umbilical cord-derived mesenchymal stromal/stem cells: In vitro study. Stem Cell Res Ther 7: 46, 2016.

16. Gapizov SS, Petrovskaya LE, Shingarova LN, Svirschevskaya EV, Dolgikh DA and Kirpichnikov MP: The effect of TNF and VEGF on the properties of Ea.hy926 endothelial cells in a model of multi-cellular spheroids. Acta Naturae 10: 34-42, 2018.

17. Wei Y, Yang Q, Zhang Y, Zhao T, Liu X, Zhong J, Ma J, Chen Y, Zhao C and Li J: Plumbagin restrains hepatocellular carcinoma angiogenesis by suppressing the migration and invasion of tumor-derived vascular endothelial cells. Oncotarget 8: 15230-15241, 2017.

18. Ladizinski B and Federman DG: Trousseau syndrome. CMAJ 185: 1063, 2013.

19. Livak KJ and Schmittgen TD: Analysis of relative gene expression data using real-time quantitative PCR and the 2(-Delta Delta C(T)) method. Methods 25: 402-408, 2001.

20. Rehmsmeier M, Steffen P, Hochsmann M and Giegerich R: Fast and effective prediction of microRNA/target duplexes. RNA 10: $1507-1517,2004$.

21. Witkos TM, Koscianska E and Krzyzosiak WJ: Practical aspects of microRNA target prediction. Curr Mol Med 11: 93-109, 2011.

22. Peterson SM, Thompson JA, Ufkin ML, Sathyanarayana $P$, Liaw L and Congdon CB: Common features of microRNA target prediction tools. Front Genet 5: 23, 2014.

23. Li J, Xia L, Zhou Z, Zuo Z, Xu C, Song H and Cai J: MiR-186-5p upregulation inhibits proliferation, metastasis and epithelial-to-mesenchymal transition of colorectal cancer cell by targeting ZEB1. Arch Biochem Biophys 640: 53-60, 2018.

24. Su BB, Zhou SW, Gan CB and Zhang XN: MiR-186 inhibits cell proliferation and invasion in human cutaneous malignant melanoma. J Cancer Res Ther 14 (Suppl): S60-S64, 2018. 
25. Thodiyil PA and Kakkar AK: Variation in relative risk of venous thromboembolism in different cancers. Thromb Haemost 87: 1076-1077, 2002.

26. Lambert EH, Pierorazio PM, Shabsigh A, Olsson CA, Benson MC and McKiernan JM: Prognostic risk stratification and clinical outcomes in patients undergoing surgical treatment for renal cell carcinoma with vascular tumor thrombus. Urology 69: 1054-1058, 2007.

27. Karnes RJ and Blute ML: Surgery insight: Management of renal cell carcinoma with associated inferior vena cava thrombus. Nat Clin Pract Urol 5: 329-339, 2008.

28. Lam JS, Klatte T, Kim HL, Patard JJ, Breda A, Zisman A, Pantuck AJ and Figlin RA: Prognostic factors and selection for clinical studies of patients with kidney cancer. Crit Rev Oncol Hematol 65: 235-262, 2008.

29. Bex A, Van der Veldt AA, Blank C, Meijerink MR, Boven E and Haanen JB: Progression of a caval vein thrombus in two patients with primary renal cell carcinoma on pretreatment with sunitinib. Acta Oncol 49: 520-523, 2010.

30. Harshman LC, Srinivas S, Kamaya A and Chung BI: Laparoscopic radical nephrectomy after shrinkage of a caval tumor thrombus with sunitinib. Nat Rev Urol 6: 338-343, 2009.

31. Karakiewicz PI, Suardi N, Jeldres C, Audet P, Ghosn P, Patard JJ and Perrotte P: Neoadjuvant sutent induction therapy may effectively down-stage renal cell carcinoma atrial thrombi. Eur Urol 53: 845-848, 2008.

32. Shuch B, Riggs SB, LaRochelle JC, Kabbinavar FF, Avakian R, Pantuck AJ, Patard JJ and Belldegrun AS: Neoadjuvant targeted therapy and advanced kidney cancer: Observations and implications for a new treatment paradigm. BJU Int 102: 692-696, 2008.

33. Hakenberg OW: Comment on Di Silverio et al: Neodajuvant therapy with sorafenib in advanced renal cell carcinoma with vena cava extension submitted to radical nephrectomy. Urol Int 2008;80:451-453. Urol Int 80: 454, 2008.
34. Roberts E, Cossigny DA and Quan GM: The role of vascular endothelial growth factor in metastatic prostate cancer to the skeleton. Prostate Cancer 2013: 418340, 2013.

35. Yan JJ, Zhang YN, Liao JZ, Ke KP, Chang Y, Li PY, Wang M, Lin JS and He XX: MiR-497 suppresses angiogenesis and metastasis of hepatocellular carcinoma by inhibiting VEGFA and AEG-1. Oncotarget 6: 29527-29542, 2015.

36. Zhao X, Mohan R, Özcan S and Tang X: MicroRNA-30d induces insulin transcription factor MafA and insulin production by targeting mitogen-activated protein 4 kinase 4 (MAP4K4) in pancreatic beta-cells. J Biol Chem 287: 31155-31164, 2012.

37. Lewis BP, Burge CB and Bartel DP: Conserved seed pairing, often flanked by adenosines, indicates that thousands of human genes are microRNA targets. Cell 120: 15-20, 2005.

38. Chen K and Rajewsky N: The evolution of gene regulation by transcription factors and microRNAs. Nat Rev Genet 8: 93-103, 2007.

39. Zhang ZL, Bai ZH, Wang XB, Bai L, Miao F and Pei HH: miR-186 and 326 predict the prognosis of pancreatic ductal adenocarcinoma and affect the proliferation and migration of cancer cells. PLoS One 10: e0118814, 2015.

40. Lee YH, Kim SY and Bae YS: Upregulation of miR-760 and miR-186 is associated with replicative senescence in human lung fibroblast cells. Mol Cells 37: 620-627, 2014.

41. Sun P, Hu JW, Xiong WJ and Mi J: miR-186 regulates glycolysis through Glut1 during the formation of cancer-associated fibroblasts. Asian Pac J Cancer Prev 15: 4245-4250, 2014.

42. Cui G, Cui M, Li Y, Liang Y, Li W, Guo H and Zhao S: MiR-186 targets ROCK1 to suppress the growth and metastasis of NSCLC cells. Tumour Biol 35: 8933-8937, 2014. 